\title{
3D Digitizing Device Applied in Evaluation and Simulation of Postoperative Trunk Surface Shape in Adolescent Idiopathic Scoliosis
}

\author{
Farida CHERIET ${ }^{\mathrm{a}, \mathrm{c}}$, Li SONG*b , Philippe DEBANNÉ ${ }^{\mathrm{a}, \mathrm{c}}$, Olivier DIONNE ${ }^{\mathrm{a}}$, Hubert LABELLE ${ }^{\mathrm{c}, \mathrm{d}}$ \\ ${ }^{a}$ Department of Computer Engineering, École Polytechnique de Montréal, Montréal (QC), Canada; \\ ${ }^{b}$ Creaform Inc., Lévis (QC), Canada; \\ ${ }^{\mathrm{C}}$ Research Center, CHU Sainte-Justine, Montréal (QC), Canada; \\ ${ }^{d}$ Department of Surgery, Faculty of Medicine, Université de Montréal, Montréal (QC), Canada.
}

\begin{abstract}
Adolescent idiopathic scoliosis (AIS) is a complex 3D deformation of the musculo-skeletal system of the trunk, with a prevalence of about $1 \%$ to $3 \%$ in the general population. Scoliosis is clinically apparent by observing the asymmetry of spinous processes, ribs, and scapulae, imbalance between the top and bottom of the spine, and left-right asymmetry of the trunk. Among patients with AIS, about 1 in 1,000 will need surgery using spinal instrumentation and fusion to correct the deformity. However, while the surgeon's main goals are to correct the spinal deformity and achieve spinal balance, the most important outcome for patients is the correction of the external shape of the trunk.

This paper provides an overview of work done in recent years by our research group to exploit data collected using a Creaform surface digitizing setup to study the surgical correction of trunk external shape of AIS patients treated at Sainte-Justine University Hospital Center (CHU). We first describe our surface acquisition system and clinical setup. We then introduce a set of clinical measurements (indices) based on the trunk's external shape, to quantify its degree of asymmetry. We then present the results of a preliminary study assessing the effect of scoliosis surgery on the external trunk shape. We finally present a hybrid, deformable model of the human trunk for prediction of surgical outcome on trunk shape in AIS.

The longer term aim of this research is to develop a validated simulation tool that would allow the clinician to illustrate to the patient the potential result of the surgery and would help in deciding on a surgical strategy that could most improve their external appearance.
\end{abstract}

Keywords: 3D body digitizing, scoliosis, 3D analysis, non-invasive data, surgery correction, simulation.

\section{Introduction}

Scoliosis is a three-dimensional deformity of the spine and the rib cage, leading to a general asymmetry of the trunk. The most frequent type of scoliosis, idiopathic scoliosis, has no specific identifiable cause. The onset of the spinal deformation is variable and becomes more prominent during the adolescent growth spurt. Scoliosis is clinically apparent by observing the asymmetry of spinous processes, ribs, and scapulae, imbalance between the top and bottom of the spine, and left-right asymmetry of the trunk in forward bending [1]. Among the adolescent population, the incidence of adolescent idiopathic scoliosis (AIS) is $4.5 \%$. Among patients with AIS, $8 \%$ to $9 \%$ will be treated by brace and about 1 in 1,000 will need surgery using spinal instrumentation and fusion to correct the deformity. In the absence of treatment, it may lead to postural problems, and even cardiac or pulmonary complications.

Usually, the evaluation of scoliosis relies on frontal and lateral radiographs, on which several 2D measurements are computed. Most importantly, the Cobb angle, defined as the angle between the end plates of the two most tilted vertebrae along the spinal curve, represents a scoliosis severity index when measured in the frontal plane. Nevertheless, in the past fifty years, many studies have documented the three-dimensional aspect of scoliosis and emphasized the importance of a 3D evaluation [2].

X-rays only reveal the spinal deformity, while the surface trunk asymmetry associated with AIS has been shown to be the most important factor affecting the patient's mental and social well-being [3,4]. A meta-analysis of the literature has demonstrated that the correction of the apparent deformity in idiopathic scoliosis was a major factor of satisfaction for patients who have had an operation [5].

\footnotetext{
* Isong@creaform3d.com; +1-418-833-4446; www.creaform3d.com
} 
Therefore, it would be useful to have an objective quantitative tool to evaluate and document the three-dimensional postoperative correction of the trunk external asymmetry. In this perspective, several methods to quantify scoliosis deformity from back or trunk surface asymmetry have been introduced since the 1970 s $[3,6,7,8,9,10,11]$. Our research team has for several years utilized non-invasive surface topography to acquire the whole trunk of patients at the Sainte-Justine University Hospital Center (CHU) scoliosis clinic in Montreal, Canada [12]. Several trunk shape indices have been developed and their reliability evaluated on patients in standing position [13].

The purpose of this paper is to provide an overview of ongoing research carried out by our group to evaluate post-operative trunk surface shape in AIS patients having undergone spinal surgery at $\mathrm{CHU}$ Sainte-Justine. This work exploits a non-invasive surface digitizing system from Creaform Inc. to scan the pre- and post-operative trunk shapes of patients during their visits to the hospital's orthopaedic clinic. Section 2 will describe our setup for acquisition of the whole trunk shape. Section 3 will introduce a set of clinical measurements (indices) based on the trunk's external shape, to quantify its degree of asymmetry, as well as the results of a preliminary study assessing the effect of scoliosis surgery on the external trunk shape using those indices. Section 4 will present a numerical model for prediction of surgical outcome on trunk shape. Finally, Section 5 will summarize the results of the studies presented here and briefly discuss its potential benefits for medical practice.

\section{Clinical setup for acquiring patient trunk surface}

\subsection{Surface digitizers}

A non-invasive system allowing the acquisition and reconstruction of the 3D trunk surface geometry of spinal deformity patients was installed and has been in routine use at CHU Sainte-Justine since 2002. The system is located in a closed room in the orthopedics clinic wing, and is comprised of four serial digitizers (Capturor II LF, Creaform Inc.) [14] as seen in Figure 1. The four digitizers are arranged in such a way as to provide maximal coverage of the trunk surface. In sequence, the different units capture the back, the front, the left side and the right side of the patient. The two lateral units are angled obliquely (approx. $50^{\circ}$ on either side of the front unit's line of sight) and are placed slightly lower than the front and rear units, in order to minimize obstruction by the patient's arms. Each digitizer is fixed to a vertical support which allows for height adjustment. All four units are placed at a given standoff distance $(1500 \pm 100 \mathrm{~mm})$ from the center of the digitizing space, at which the patient must stand.

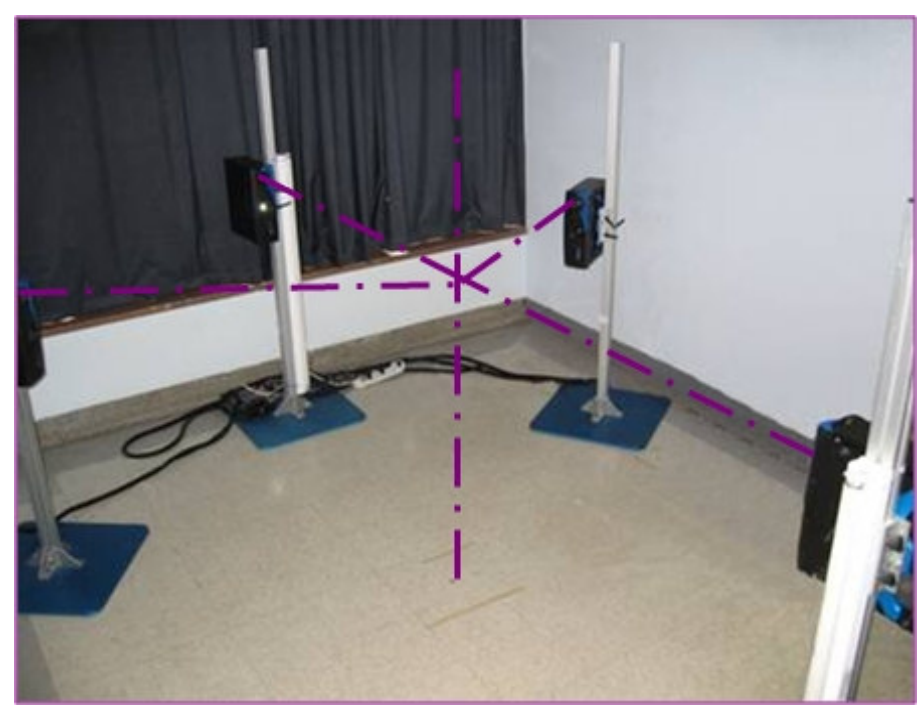

Fig. 1. Clinical setup of four 3D optical digitizers (Creaform Inc.).

Each digitizer consists of a halogen structured light projector (white light source and grating slide) combined with a standard color CCD camera of resolution $1024 \times 768$ pixels. A single acquisition comprises five images: four with phase-shifted fringes for measuring the geometry (using the interferometry principle combined with active triangulation) and one without fringes to get the surface texture data. The acquisition time for each digitizer is slightly over one second, and the total time for all four units to shoot in sequence is around five seconds. A software package provided with the digitizing system, FAPS, is used to control the acquisition process. It is then used to process the raw camera images to obtain a phase function, then to unwrap the phase function and convert the resulting depth 
map into a metrical surface with texture mapped. Figure 2 illustrates this process in the case of the back of an anthropomorphic manikin.

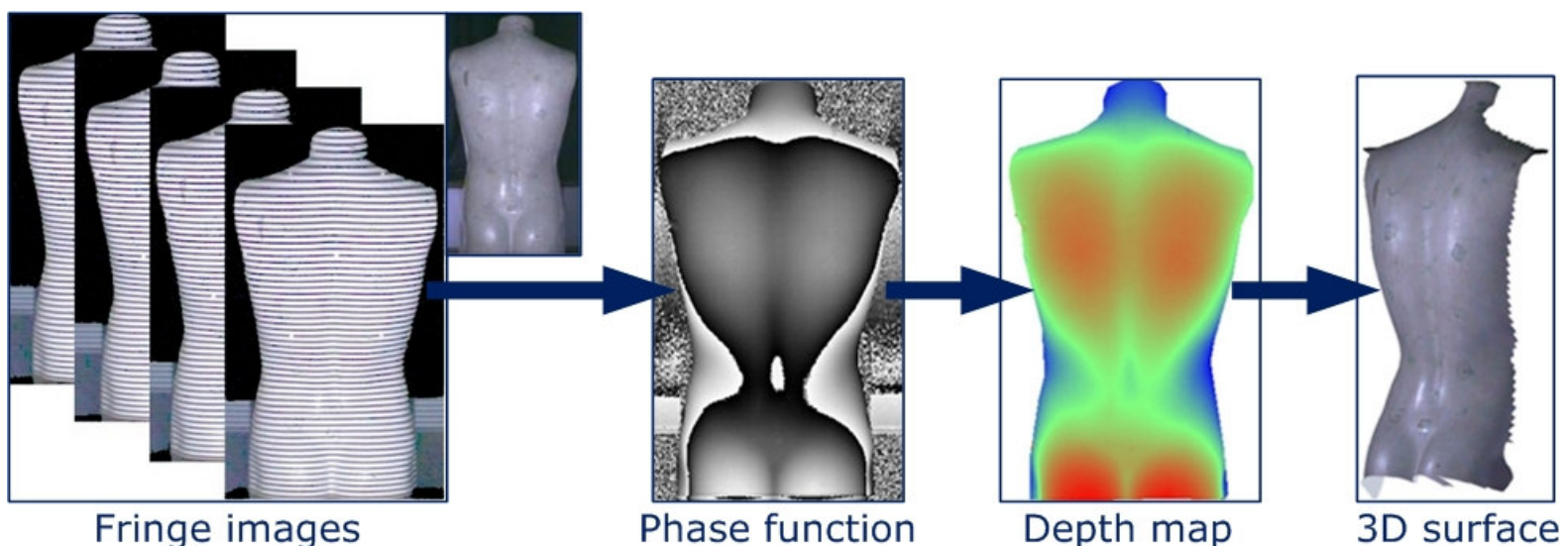

Fig. 2. Steps to process the raw digitizer images (4 fringe +1 texture).

To obtain the geometry of the entire trunk, the partial surfaces acquired by the four digitizers must be registered and merged. Here, a second software package also provided with the system, EM, is used to import the partial surfaces produced in FAPS, register them into a common reference frame and merge the geometries and textures into a single model, as illustrated by Figure 3 . In order to register the partial surfaces properly, a multi-head calibration, exploiting a known planar reference target visible to all the cameras, is performed on a regular basis. The reconstructed surface region does not include the arms or the head. The resulting surface is a textured, high-density polygonal mesh. For an entire trunk of average size, the reconstructed polygonal mesh numbers around 180000 vertices. For easier manipulations, the data is usually sub-sampled with the result counting, depending of the patient's height, from 40000 to 70000 points. The EM program allows the complete model to be exported in a variety of file formats to facilitate subsequent analysis with our team's own in-house tools.

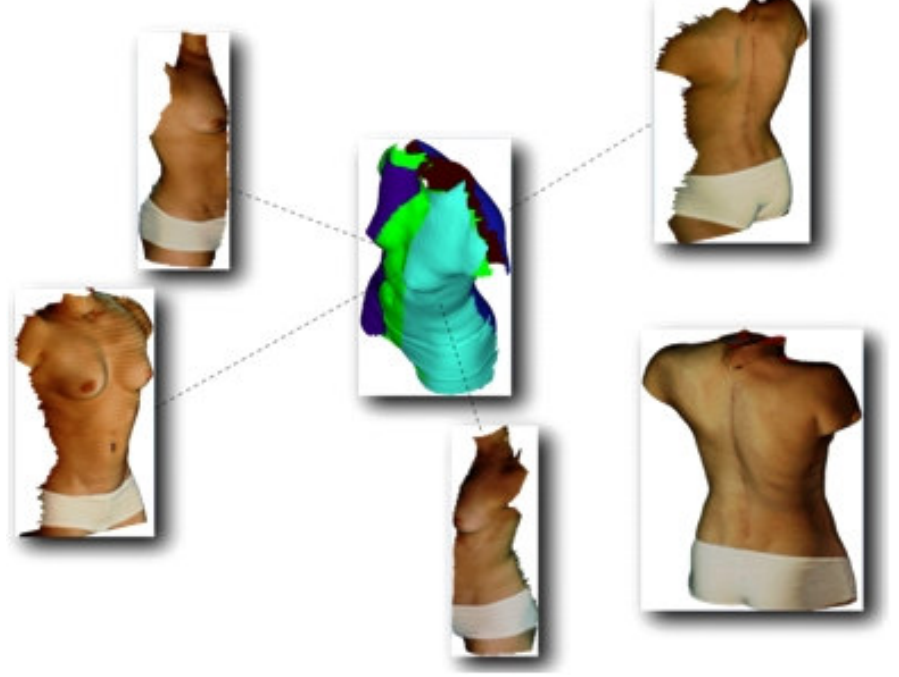

Fig. 3. Registration and merging of partial surfaces of human trunk.

\subsection{Acquisition protocol}

Candidates for scoliosis surgery at CHU Sainte-Justine are scheduled to have surface topography scans as part of their preoperative clinical visit. At the preoperative visit, the patient is scanned in neutral standing position. Before surface acquisition, the patient is asked to remove his or her upper garments, down to the waist level. Then, the technician places visible markers on the patient's skin, indicating: left and right ASIS, spinous processes of the prominent vertebra, T1, apex and limit vertebrae, sternum extremities, clavicle extremities, hip extremities and several other landmarks on the rib cage.

The subject must stand at the center of the system (marked on the floor) with shoes removed and with any long hair tied up over the neck. He/she is asked to stand still during acquisition, focusing on a point 
above the front digitizer. The neutral standing posture is similar to the anatomical posture adopted by many clinicians, with the arms in slight abduction by the sides. The technician explains the test and demonstrates it to the subject prior to the acquisition. Figure 4 shows a preoperative patient seen from the back, with the fringe pattern projected. The test is repeated a second time; between each acquisition, the subject must relax for at least half a minute, corresponding to the time needed to save the data, before repositioning. In subsequent processing, if the first trial proves to be inadequate (due to excessive patient movement, for instance), then the second trial can be retained instead for subsequent analysis.

After surface acquisition but prior to X-rays acquisition, radio-opaque markers are placed over the previous ones. These corresponding markers are used to perform elastic registration of the internal and external geometries, which are acquired non-simultaneously and with the patient in possibly varying postures.

After the spinal surgery, the same patient is scheduled to return to the orthopaedic clinic for follow-up visits, usually at six months, one year and two years after the surgery date. At these visits, trunk surface topography is again acquired in the manner described above.

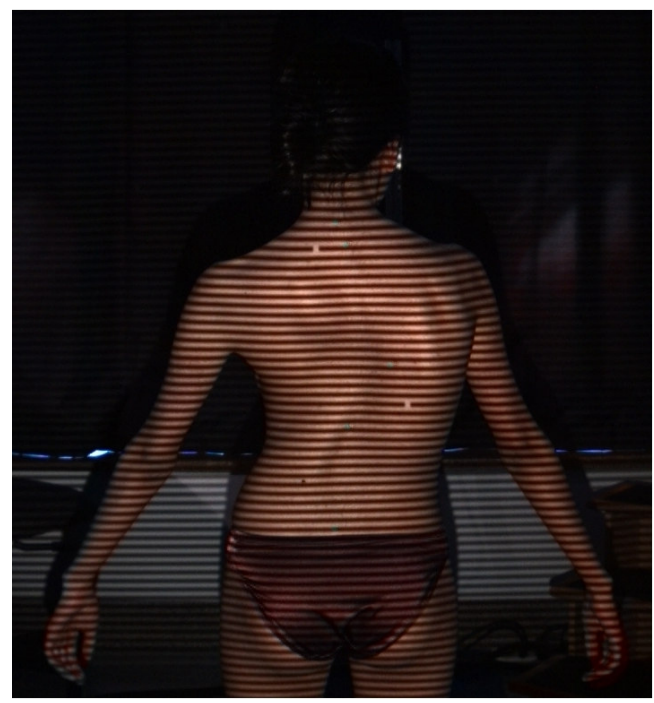

Fig. 4. Typical acquisition of patient at preoperative visit: standing (with fringes projected).

\subsection{Accuracy assessment}

A previous study of this system was carried out in order to assess the accuracy of this surface topography system in reconstructing the human trunk [12]. For this purpose, repeated acquisitions and reconstructions were performed on an inanimate anthropomorphic manikin (seen in Figure 2 above). In order to have a dataset of 3D points to compare between measurement modalities, 39 markers were fixed on the manikin's trunk, some over anatomical landmarks, the others distributed so as to cover the entire trunk. Each marker consisted of a wire cross with an intersection in a sphere $1.3 \mathrm{~mm}$ in diameter. The 3D coordinates of these intersections were measured with a coordinate measuring machine $(\mathrm{CMM})$, having accuracy on the order of a few microns. These CMM measurements served as the reference data.

A total of 12 reconstructions of the manikin were built from three series of four acquisitions. For each trial, two sets of data were then compared with the reference data: first, he reconstructed polygonal surface of the trunk, and second, the positions of the markers measured by 3D selection on the textured surface of the 3D reconstruction. In the first test, the reference point set was registered to the 3D surface by minimizing the average normal distance of the points to their closest surface polygons (point-to-surface registration). In the second test, the reference point set was registered to the corresponding selected points from the textured 3D surface using an algorithm based on the singular value decomposition method (point-to-point registration).

For the point-to-surface registration, the average normal distance between the reconstructed surfaces and the reference data was estimated at $1.1 \pm 0.9 \mathrm{~mm}$ over the entire set of markers. For the point-to-point registration, the errors were normally distributed with a standard deviation of $1.8 \mathrm{~mm}$ in the horizontal lateral direction and $1.6 \mathrm{~mm}$ in the vertical direction; for the direction of the depth co-ordinate, the standard deviation was $1.6 \mathrm{~mm}$. Overall, the errors were normally distributed with a standard deviation of $1.4 \mathrm{~mm}(n=468)$. These results are of the same order of magnitude as the 
digitiser resolution and as the marker thickness, and represent small errors compared to the variability of manually placing markers over the anatomical landmarks of the human trunk. Analysis revealed several factors having an influence on reconstruction precision: lighting conditions (to be expected when ambient light is too strong or using fluorescent ambient light), region of the trunk surface (our specific setup yielding the best data quality for the back portion, the accuracy being approximately 0.5 $\mathrm{mm}$ in that region), and registration of the partial surfaces to form the complete geometry (which depends on the quality of the multi-head calibration). In conclusion, the accuracy of the 3D reconstruction system was found to be adequate for analysis of human trunk asymmetry.

\section{Indices describing external trunk shape}

Since the 1970s, several different systems have been used to acquire the surface shape of the back or whole trunk of scoliotic patients, and in that context, indices to quantify asymmetry, both locally in terms of measures from surface cross-sections and globally in terms of measures from surface regions, have been developed by various research groups $[3,6,7,8,9,10,11]$. Each new technology introduces a different approach to understanding scoliosis via a different set of data describing the spine or the trunk. The challenge for the evaluation of surface asymmetry comes from the precision and accuracy needed, considering that the goal is to be able to detect and quantify small but significant changes of the trunk surface. And contrary to the assessment of scoliotic deformities from X-ray images where the Cobb angle is the gold standard, there is no consensus yet on a set of indices that can be used to assess these deformities from the trunk surface. Our own team has developed several trunk shape indices, and we will focus here on those that are computed locally on cross-sections extracted from the trunk shape.

\subsection{Extraction of trunk cross-sections}

Once the polygonal textured model of the whole trunk is obtained, the initial step is to identify a set of landmarks previously marked on the skin: mainly, the anterior-superior iliac spines (ASIS), vertebra prominens (VP), the midpoint of the posterior-superior iliac spines (PSIS), and the spinous processes of T1 and of the apex and limit vertebrae of the scoliotic curve(s) (identified along the back valley). A patient-specific reference frame is then defined as follows: the origin is located at the PSIS midpoint (i.e. on the patient's back at the pelvic level); the $X$ axis points to the right of patient, its direction given by the line passing through the two ASIS projected on the horizontal plane; the $Z$ axis points toward the back; and the $Y$ axis points upward.

A set of horizontal (parallel) cross-sections is computed as the intersections of the set of horizontal cutting planes with the polygonal mesh. In order to have a smooth, uniformly spaced and ordered set of points forming a closed contour for each cross-section, an interpolating or approximating spline curve is fitted to the intersection points and sampled to obtain a fixed number of uniformly spaced points. If $n=250$ cross-sections are thus generated between the PSIS level and the T1 level, this represents a vertical spacing of between 2 and $3 \mathrm{~mm}$ depending on the trunk height.

In order to analyze the cross-sections and compute indices on them, it is necessary to establish a local reference frame for each section. The idea is to determine the major direction of variation of the coordinates of the set of points forming a section, which will be inscribed in the plane of the section itself. This defines the major axis of the section, with a minor axis perpendicular to it. A simple means of determining the major axis is by least squares approximation of the depth ( $Z$ ) coordinates of the points. However, a more reliable approach consists in a principal component analysis on the 3D positions of the points to extract the major and minor axes, which define respectively the $\mathrm{X}$ and $\mathrm{Z}$ axes of the section's local reference system, the $Y$ axis being normal to the other two and pointing upward. The origin is located at the centroid, i.e. the geometric center of the section (see Figure 5).

\subsection{Indices based on cross-sections}

Two complementary indices are frequently used in our analyses: the trunk rotation (TR) and the back surface rotation (BSR). These are defined as follows:

- Trunk Rotation (TR): angle between the section's local $X$-axis and the patient $X$-axis; this angle is signed, with positive angles for clockwise rotation around $Y$ and negative angles otherwise.

- Back Surface Rotation (BSR): angle between the dual tangent to the back profile and the patient $\mathrm{X}$-axis; this angle is also signed, with the sign having the same meaning as for the trunk rotation. 
Figure 5 illustrates these two definitions. Note that the dual tangent to the back profile is the straight line that is tangential to (i.e. just touching) the left and right ribcage humps on the posterior profile. These humps can be found by searching for the local maxima of the back profile (zeros of the first derivative). Each measure is computed over the set of $n$ sections, thus yielding a scalar function of either the height along the trunk ( $\mathrm{Y}$ coordinate in patient frame) or of the section number.
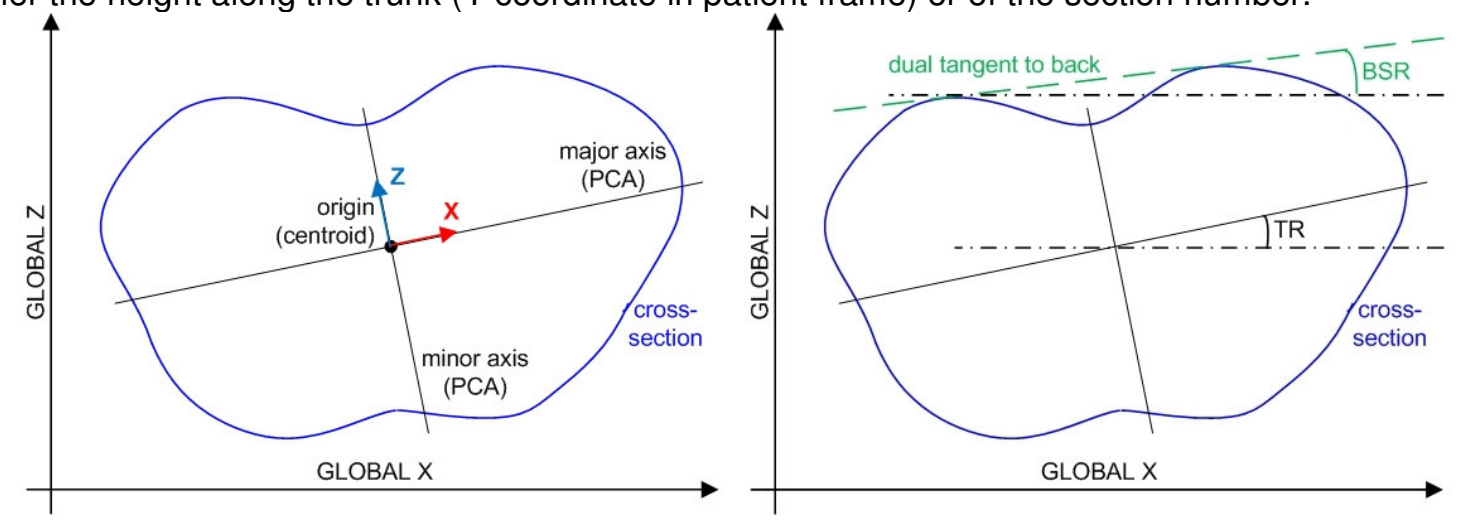

Fig. 5. Graphical definitions of the section local reference frame and the TR and BSR indices.

These two indices seem similar in their definition, but they do not measure exactly the same characteristics. While the BSR only exploits the back portion of a given section, the TR also includes the anterior profile that contributes to the perception of cosmetic deformation. Thus, these two measurements are complementary, in that the TR captures both the rotation of the section with respect to the patient frame and its overall deformation, while the BSR captures the local gibbosity of the back. With a combined interpretation of both measures, it is possible to differentiate the axial rotation of transverse sections from their deformations. Together, they provide a more adequate characterization of the rib cage deformity.

\subsection{Reliability assessment}

Our team previously undertook to estimate the reliability of a set 3D trunk surface measurements for the characterization of external asymmetry associated with scoliosis [13]. Repeated trunk surface acquisitions using the Creaform system, with two different postures A (anatomical position, with arms in slight abduction) and B ("clavicle" position, with elbows raised forward and hands on the sides of the neck), were obtained from a cohort of 49 AIS patients who attended the CHU Sainte-Justine scoliosis clinic. For each acquisition, a 3D model of the patient's trunk was built and a series of measurements was computed. These measurements included the cross-section based TR and BSR indices as defined above, with their maximal values in absolute value over the whole trunk being retained for analysis. This study also looked at several global indices, namely trunk inclination, pelvic tilt, shoulders imbalance and apparent global asymmetry. The global measurements use the manually identified 3D coordinates of anatomical landmarks as in section 2.2. The apparent global asymmetry also uses the series of left and right posterior humps detected on the cross-sections.

For each measure and posture, intraclass correlation coefficients (ICC) were obtained using a bivariate analysis of variance, and the smallest detectable difference was calculated. For posture A, reliability was fair to excellent with ICC between 0.91 and 0.99 , while for posture B, the ICC was between 0.85 and 0.98 . The smallest statistically significant differences for the maximal BSR was $2.5^{\circ}$ and $1.5^{\circ}$ for the maximal TR. The within-subject standard deviation for the maximal BSR was $1.4^{\circ}$ and $0.8^{\circ}$ for the maximal TR. Apparent global asymmetry and trunk rotation indices were relatively robust to changes in arm posture, both in terms of mean values and within subject variations, and also showed a good reliability. For the arms positioning, the anatomical posture was slightly more reliable as it allowed a better coverage of the trunk surface by the digitizing system.

Computing measurements from cross-sectional analysis enabled a reduction in errors compared to the measurements based on markers' positions. Visual examination of the cross-sectional indices in individual cases revealed that the BSR was prone to significant variability and presence of outliers in the upper thoracic region. The TR, on the other hand, tended to minimize the amplitude of the apparent deformity and had a better reliability than the BSR (ICC $=0.97 \mathrm{vs}$. ICC $=0.93)$, partly because it was less sensitive to the noise from the scapula borders.

Although not yet sensitive enough to detect small changes for monitoring of curve natural progression, trunk surface analysis can nevertheless document the external asymmetry associated with different types of spinal curves as well as the cosmetic improvement obtained after surgical interventions. 


\subsection{Assessment of trunk post-operative asymmetry correction}

A secondary aim of the study presented in [13] was to evaluate the feasibility of applying the external surface measurements to quantifying the effect of spinal instrumentation on cosmetic appearance. To do so, two exemplar AIS cases having undergone surgery were selected; for both patients, the Cobb angle was $74^{\circ}$ prior to surgery and $16^{\circ}$ afterwards. Figure 7 shows photos of their backs before and after the surgery. External surface measurements were computed and compared; transverse decomposition of the trunk used 250 horizontal cross-sections.
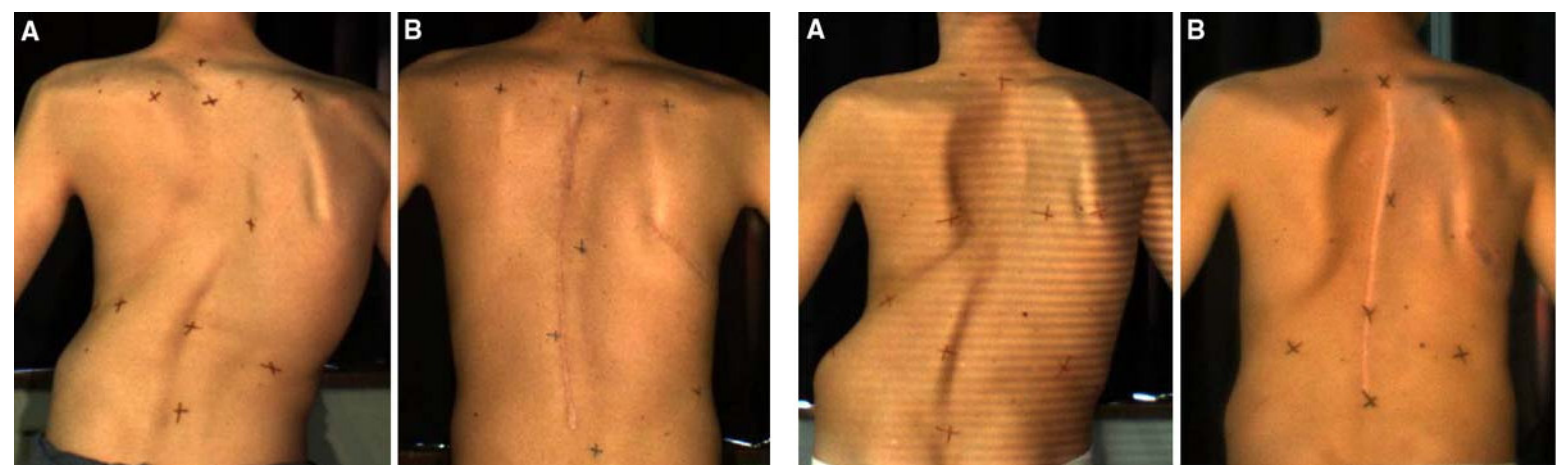

Fig. 7.Two cases of AIS before (A) and after (B) spinal surgery: patient 1 (left) and patient 2 (right).

Statistically, the preoperative external asymmetries were significantly different for most of the measurements. Whereas both cases showed good correction in the frontal plane, the ranges of corrections in other planes were not comparable. The change in the shape of the cross-sectional sections was particularly representative of the overall correction. Indeed, patient 2 showed better correction of the max. BSR and max. TR values (respectively $8.1^{\circ}$ and $6.2^{\circ}$ of correction) than patient 1 (respectively $1.8^{\circ}$ and $2.6^{\circ}$ of correction). This particular result is in keeping with a visual observation of the two patients' cosmetic improvements, which can be done by examining Figure 7.

\section{Simulation of trunk post-operative shape correction}

Spinal surgery for scoliosis consists in rectifying the spine shape using metal rods anchored to the vertebrae by means of screws and hooks. The prediction of surgical outcome is a fundamental element of any preoperative evaluation. Currently, the clinical method to define a surgical strategy and estimate the result of curve correction relies primarily on radiographic analysis of spinal flexibility and on the surgeon's own experience. To further assist the clinician during surgical planning, a biomechanical simulator is currently being developed at CHU Sainte-Justine to identify the optimal configuration of the implants to best correct the spinal deformities [15]. However, neither this simulator nor the spinal flexibility analysis consider the soft tissues of the trunk in order to provide information on the patient's external appearance after the intervention. For the surgeon, the residual trunk asymmetry proves highly subjective and his experience remains his only asset. This is problematic considering that the main reason to prescribe an operation comes initially from the patient's dissatisfaction with their apparent deformity. At present, there is no tool available to estimate the effect of treatment on the patient's external appearance, even though surgeons' assessments of treatment outcomes are not significantly correlated with patient satisfaction [16].

Therefore, the goal of a recent project was to develop a simplified physical model of the deformable tissues between the skin surface (epidermis) and bone structures of the trunk in order to visualize in $3 \mathrm{D}$ and assess the effect of scoliosis surgery on the patient's external appearance [17]. This research focused only on the soft tissues since biomechanical modeling of the bone structures is the subject of another ongoing project. Consequently, an expected postoperative configuration of the bone structures served as our basis to predict the external appearance after scoliosis surgery.

To achieve our goal, we first proposed a methodology to build a simplified system to model the different deformable structures of the trunk. Initially, 3D pre and postoperative reconstructions of the bone structures were obtained from standard radiographs while non-invasive 3D optical digitizers acquire the external surface of the trunk using white non-ionizing structure light. Following certain mesh pre-processing, we developed a generic method to generate three different tetrahedral layers starting from the external surface of the trunk to represent the skin, fat and muscles. From these new layers, a generalized particle system based on elastic potential energy was defined. Forces preserving distance, area and volume constraints were calculated to describe the physical behavior of the various soft tissues. Finally, a rigid articulated model of the bone structures was created in order to transform 
the internal preoperative configuration to the postoperative state. By solving a set of dynamic equations, the displacements of this rigid model deform the simplified soft tissue layers of the trunk in order to predict the external appearance after scoliosis surgery.

We validated this system by comparing the simulated and actual postoperative trunk surface shapes of an AIS patient having undergone surgery. For this purpose, clinical indices of torso asymmetry were computed and compared by using cross-sections of the simulated and acquired postoperative external geometries at various vertebral levels. A preliminary evaluation study for this patient showed a mean absolute error of $1.38^{\circ}$ in the thoracic region and $3.26^{\circ}$ in the lumbar region on the BSR index while the mean absolute error on the rib hump index (difference between left and right posterior rib humps, as a distance) was evaluated at $2.73 \mathrm{~mm}$ (thoracic) and $3.83 \mathrm{~mm}$ (lumbar). Figure 9 shows the simulated and actual post-op trunk shapes for the exemplar patient.

This project, has allowed us to demonstrate the feasibility of simulating the external trunk appearance resulting from corrective scoliosis surgery. A software prototype allowing a user to interactively simulate the effect of scoliosis surgery on the external trunk appearance was also developed in this project (see Figure 9). However, our results reveal the limits of the simplified modeling framework. Improved accuracy of simulation results would require the development of a more refined meshing of the different structures of the human trunk including calibrated physical properties.
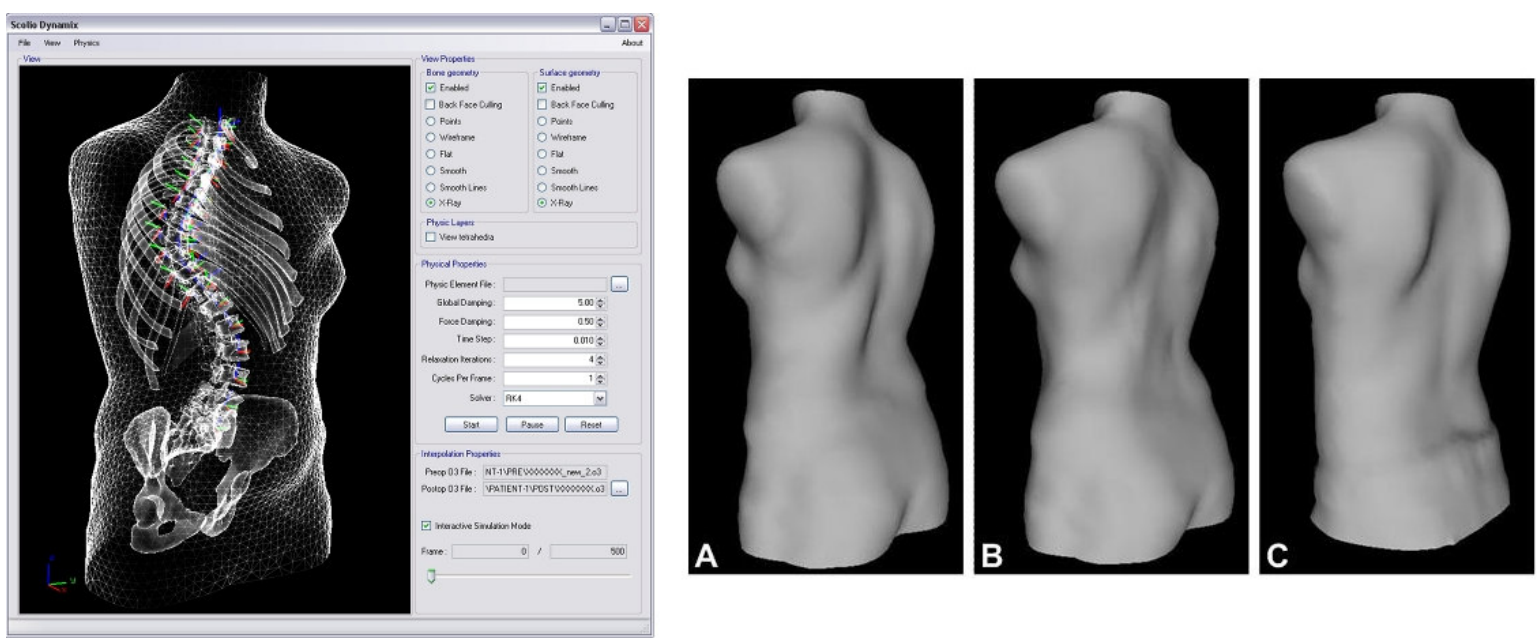

Fig. 9. Left: Software GUI for controlling physical model simulation. Right: Sample result of a simulation on the external trunk shape: A) real pre-op trunk shape; B) simulated post-op trunk shape; C) real post-op trunk shape.

\section{Summary}

In summary, we have presented in this article some of our research projects at CHU Sainte-Justine and École Polytechnique de Montréal making use of non-invasively acquired surface topography of the human trunk to study trunk asymmetry and the effects of corrective surgery in adolescent idiopathic scoliosis, and to simulate the effect of surgery on trunk external shape.

\subsection{Future work}

Several avenues for future work present themselves to improve and further validate the methodologies and results presented in this paper. Firstly, we aim to carry out the evaluation of trunk post-operative asymmetry correction on a prospective cohort of AIS patients. This larger study will allow us to better understand the effect of the surgery on trunk shape as expressed by different asymmetry indices, as well as the external correction's relationship with the correction of underlying bone structures, i.e. the spine and ribcage.

For the simulation of trunk post-operative shape correction, the numerical model must be validated on a group of patients for whom pre- and post-surgery surface acquisitions are available. Furthermore, we will focus on constructing a personalized multi-layer geometric model of the trunk from multimodal image fusion integrating surface topography, 3D radiographic reconstruction and MRI scans. We will also explore how to best exploit a priori data to calibrate the properties of the physical model.

\subsection{Potential benefits for the healthcare system}

The proposed approach for trunk shape analysis is complementary to the standard radiographic analysis used in surgery planning and evaluation of surgical outcome. Our methodology distinguishes 
itself by evaluating what is perceived by the patient as the major problem (asymmetrical appearance and rib hump) and what is considered by patients as the most important outcome, as opposed to what surgeons currently evaluate as the main outcome with Cobb angles and sagittal and coronal balance of the spine. By the same token, it could form the basis for recommendations on surgical strategies most likely to improve the patient's external appearance.

As for the surgical simulator based on a deformable model of the trunk, the potential benefits of this system will be as follows: 1 ) it will assist the surgeon, together with the patient, in deciding on the best surgical approach based on the resulting external trunk appearance; 2) it will provide an enhanced preoperative planning tool allowing the surgeon to take into account such factors as the residual external trunk asymmetry after the operation, when deciding on a surgical strategy including the indication for rib resection or anterior spine release; 3 ) it will allow the patient to readily understand the probable outcome as well as the risks of surgery and to be involved in the decisional process; 4 ) it has potential for generalized use by orthopedic surgeons.

\section{References}

1. Reamy, B. V., Slakey, J. B., (2001): "Adolescent idiopathic scoliosis: review and current concepts", American Family Physician, Vol. 64, No.1, pp. 111-116.

2. Labelle, H., Dansereau, J., Bellefleur, C., Jéquier, J. C., (1995): "Variability of geometric measurements from three-dimensional reconstructions of scoliotic spines and rib cages", European Spine Journal, Vol. 4, No. 2, pp. 88-94.

3. Goldberg, C. J., Kaliszer, M., Moore, D. P., Fogarty, E. E., Dowling, F. E., (2001): "Surface topography, Cobb angles and cosmetic change in scoliosis", Spine, Vol. 26, pp. E55-E63.

4. Pratt, R. K., Burwell, R. G., Cole, A. A., (2002): "Patient and parental perception of adolescent idiopathic scoliosis before and after surgery in comparison with surface and radiographic measurements", Spine, Vol. 27, pp. 1543-1552.

5. Haher, T. R., Merola, A., Zipnick, R. I., et al., (1995): "Meta-analysis of surgical outcome in adolescent idiopathic scoliosis. A 35-year English literature review of 11,000 patients", Spine, Vol. 20, No. 14, pp. 1575-1584.

6. Moreland, M. S., Pope, M. H., Wilder, D. G., (1981): "Moiré fringe photography of the human body", Medical Instrumentation, Vol. 15, pp. 129-132.

7. Stokes, I. A. F., Armstrong, J. G., Moreland, M. S., (1988): "Spinal deformity and back surface asymmetry in idiopathic scoliosis", Journal of Orthopaedic Research, Vol. 6, No. 1, pp. 129-37.

8. Dawson, E. G., Kropf, M. A., Purcell, G., (1993): "Optoelectronic evaluation of trunk deformity in scoliosis", Spine, Vol. 18, pp. 326-331.

9. Theologis, T. N., Fairbank, J. C. T., Turner-Smith, A. R., (1997): "Early detection of progression in adolescent idiopathic scoliosis by measurement of changes in back shape with the integrated shape imaging system scanner", Spine, Vol. 22, pp. 1223-1227.

10. Thometz, J. G., Lamdan, R., Liu, X. C., (2000): "Relationship between Quantec measurement and Cobb angle in patients with idiopathic scoliosis", Journal of pediatric orthopedics, Vol. 20, pp. 512-516.

11. Jaremko, J. L., Poncet, P., Ronsky, J., (2002): "Indices of torso asymmetry related to spinal deformity in scoliosis", Clinical biomechanics, Vol. 17, No. 8, pp. 559-568.

12. Pazos, V., Cheriet, F., Song, L., Labelle, H., Dansereau, J., (2005): "Accuracy assessment of human trunk surface 3D reconstructions from an optical digitizing system", Medical \& biological engineering \& computing, Vol. 43, No. 1, pp. 11-15.

13. Pazos, V., Cheriet, F., Dansereau, J., Ronsky, J., Zernicke, R. F., Labelle H., (2007): "Reliability of trunk shape measurements based on 3-D surface reconstructions". European Spine Journal, Vol. 16, pp. 1882-1891.

14. Song, L., Lemelin, G., Beauchamp, D., Delisle, S., Jacques, D., Hall, E. G., (2001): "3D measuring and modeling using digitized data acquired with color optical 3D digitizers and related applications", Proceedings of 12th Symposium on 3D technology, Yokohama, Japan, pp 59-77.

15. Aubin, C.-E., Labelle, H., Ciolofan, O., (2007): "Variability of spinal instrumentation configurations in adolescent idiopathic scoliosis", European Spine Journal, Vol. 16, No. 1, pp. 57-64.

16. Buchanan, R., Birch, J. G., Morton, A. A., Browne R. H., (2003): "Do you see what I see? Looking at scoliosis surgical outcomes through orthopedists' eyes", Spine, Vol. 28, No. 24, pp. 2700-2705.

17. Dionne, O., Assi, C. K., Guibault, F., Labelle, H., Cheriet, F., (2010): "Postoperative Appearance Simulation of the External Outcome of Spine Surgery: Preliminary Results", Research into Spinal Deformities 7, C.-E. Aubin, I.A.F. Stokes, H. Labelle and A. Moreau (eds), p. 270. 